\title{
Contrast-enhanced lesions on computerised tomography in multiple sclerosis
}

\author{
A. E. H A R D I G , E. W. R A D UE, A N D A . M . W H I T L E Y
}

From The National Hospital for Nervous Diseases, Queen Square, London

SUMMARY Two patients are described in whom computerised tomography revealed contrastenhanced lesions in the early stages of multiple sclerosis. Such lesions may be differentiated from tumours by their transient nature, lack of space-occupying effect, and localisation in the white matter. Contrast enhancement in demyelinating disease is probably related to local breakdown of the blood-brain barrier.

Recent publications (Cala and Mastaglia, 1976; Gyldensted, 1976a, b; Jacobs and Kinkel, 1976; Wüthrich et al., 1976) have described a high incidence of abnormal computerised tomography (CT) scans in patients with multiple sclerosis. Low attenuation lesions situated mainly in the white matter, ventricular dilatation with irregular edges, and enlarged sulci are the most frequent findings. Contrast enhancement of demyelinating lesions appears to be rare; Wüthrich et al. (1976) reported one case in a series of 60 patients, and there is only one other in the literature (Cole and Ross, 1977). Both patients were diagnosed as having multiple sclerosis on clinical grounds. We describe two further patients with contrast-enhanced lesions in whom a definite diagnosis of demyelinating disease could not be made at the time of scanning, either clinically or with the aid of cerebrospinal fluid (CSF) examination and visual evoked potentials. In both cases the subsequent clinical course pointed to "clinically definite" multiple sclerosis (McDonald, 1975), and the diagnosis was supported by typical visual evoked potentials and CSF changes.

\section{Case reports}

CASE 1 (NATIONAL hospital NO. A82468)

A 24 year old right handed woman developed progressive weakness of the right arm and leg over a period of 12 hours, preceded by severe left sided frontal headache. She had been taking an oral contraceptive pill for six years and had suffered

Address for reprint requests: Dr A. E. Harding, Department of Neurological Science, Royal Free Hospital, Pond Street, London, NW3 2QG. Accepted 24 February $197^{\circ}$ from migraine during that time but not before. She smoked 20 cigarettes a day.

On examination two days after the onset of symptoms her speech was normal. Sensation was subjectively reduced on the right side of the faced and there was mild right facial weakness. The $\frac{?}{\mathrm{D}}$ right arm and leg were weak in a pyramidalo distribution with increased reflexes. Plantar responses were flexor and abdominal responsesô normal. All sensory modalities were reduced op the right side. General examination was negative and the blood pressure $140 / 90 \mathrm{mmHg}$.

Routine haematology and biochemistry were normal and serological tests for syphilis negative. The CSF contained 28 mononuclear cells per $\mathrm{mm}^{3}$, and the protein content was $0.39 \mathrm{~g} / 1$, IgG $7.6 \%$. Left carotid angiography was normal. The visual evoked response from the right eye had a slightly prolonged latency of $127 \mathrm{~ms}$ (normal range up to $115 \mathrm{~ms}$ ) and an abnormal waveform thought to be equally consistent with demyelination or a vascular lesion.

Over the next month the patient recovered near normal function of the right arm and leg, and she was well until one year later when her hemiparesis recurred suddenly. On this occasion she had no sensory loss but examination was otherwise identical to that of her previous admission. Repeat CSF examination showed 10 mononuclear cells per $\mathrm{mm}^{3}$, protein $0.36 \mathrm{~g} / \mathrm{l}, \mathrm{IgG} 19.5 \%$. Left carotid angiography was again normal. A gamma scan showed increased uptake in the left parietal region. A CT scan showed a poorly defined low density area (up to 8 EMI units) in the left parietal lobe (Fig. 1a). After injection of sodium iothalamate (Conray) there was marked enhancement of 
the area (Fig. 1b). This appearance was thought to be consistent with that of a glioma.

At this stage neurosurgical intervention was not thought justifiable in view of the size and position of the lesion. The patient was treated with a four week course of dexamethasone, and recovered to the extent of only noticing clumsiness of the right hand when she was drawing. Nine months later she had a three day episode of unsteadiness and falling to the left while on holiday in Greece. She was readmitted to the National Hospital one month later with a three day history of difficulty in finding words.

On examination she had a mild motor dysphasia and dysarthria. The reflexes were symmetrically very brisk but the plantar responses were flexor. The right abdominal reflexes were absent. She was mildly ataxic in all four limbs, and Romberg's test was positive.

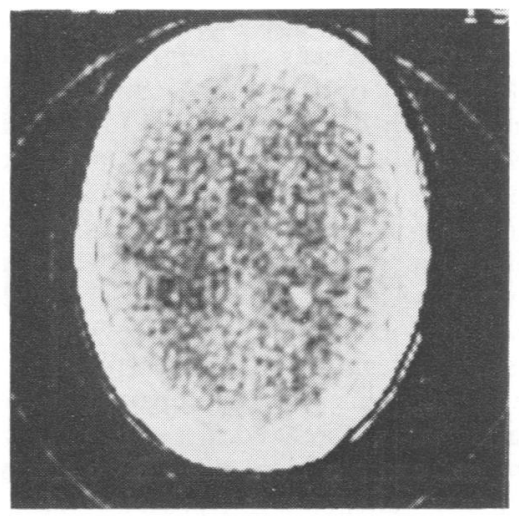

(a)

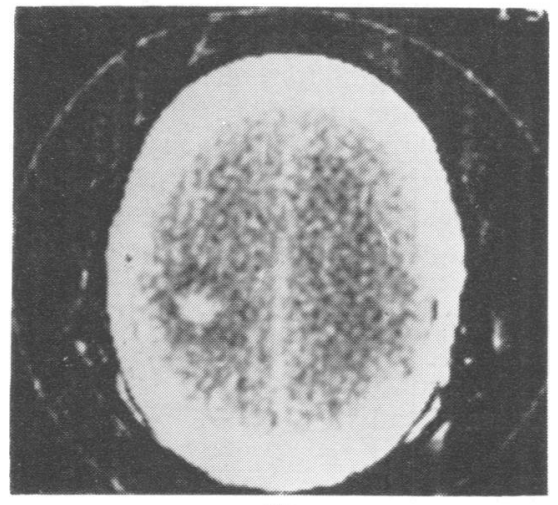

(b)

Fig. 1 Case 1. (a) Unenhanced scan showing focal low attenuation (8 EMI units) in the left parietal lobe without mass effect. (b) After intravenous sodium iothalamate: dense central enhancement.
The CSF on this occasion contained 39 mononuclear cells per $\mathrm{mm}^{3}$, protein $0.78 \mathrm{~g} / \mathrm{l}$, IgG $15.8 \%$, and an oligoclonal gamma globulin pattern typical of multiple sclerosis. Visual evoked potentials were of normal shape but the latency from the right eye was $104 \mathrm{~ms}$ compared to $95 \mathrm{~ms}$ from the left. A CT scan with enhancement was normal (Fig. 2).

The patient's speech disturbance resolved within a few days, and her ataxia improved gradually over a period of two months.

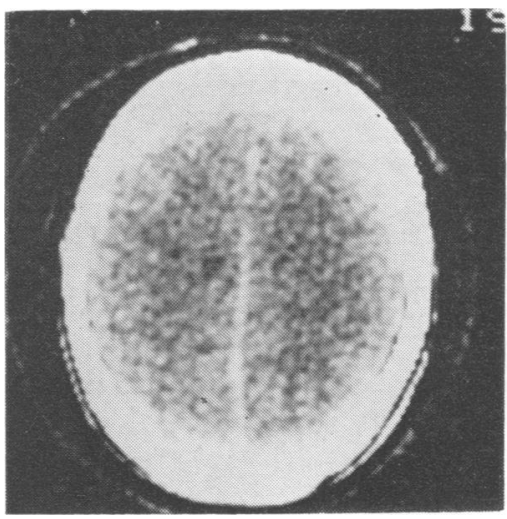

Fig. 2 Case 1. Normal enhanced scan one year later.

CASE 2 (national hospital no. A83612)

A 31 year old right handed woman was admitted to the National Hospital with a two month history of darkening of vision on the right side of her visual fields. One week before admission she had developed word finding difficulties. At the same time she had noticed problems with reading because of lack of understanding, and tended to spell incorrectly and to make simple grammatical errors when writing. She had taken an oral contraceptive pill for seven years until one month before admission.

On examination she had a moderate expressive and nominal dysphasia. Dressing was apraxic. She had mild dyscalculia, dyslexia, dysgraphia, and right-left disorientation but no finger agnosia. There was a dense right homonymous hemianopia with macular sparing. She had right facial weakness and mild right sided pyramidal type weakness in the limbs. Reflexes, co-ordination, and sensation were normal. General examination was negative.

Routine haematology and biochemistry were normal and serological tests for syphilis negative. The CSF contained one mononuclear cell per $\mathrm{mm}^{3}$, protein $0.65 \mathrm{~g} / 1, \mathrm{IgG} 9.5 \%$. Visual evoked potentials were grossly asymmetrical but not delayed. Gamma scan showed two areas of increased 
uptake in the left frontal and temporal regions. The CT scan showed a low attenuation (12 EMI units) area adjacent to the wall of the left lateral ventricle (Fig. 3a). After intravenous sodium iothalamate dense, ill-defined enhancement of four areas around the lateral ventricles was seen (Fig. 3b). Carotid angiography confirmed the presence of ventricular dilatation but no other lesion was seen.

The patient was thought to have multiple cerebral metastases but intensive investigation failed to establish a primary lesion. During admission her dysphasia improved considerably. Three weeks

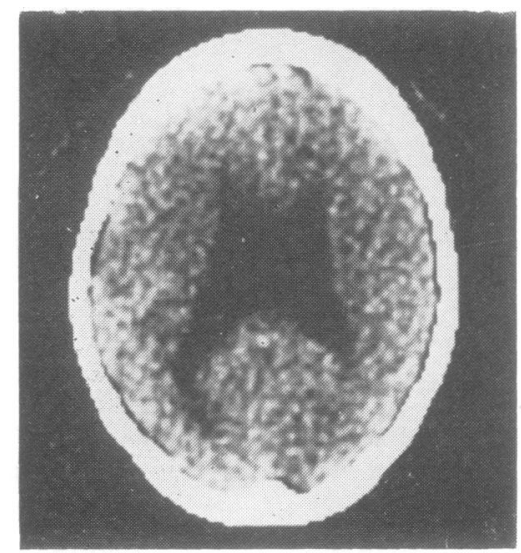

(a)

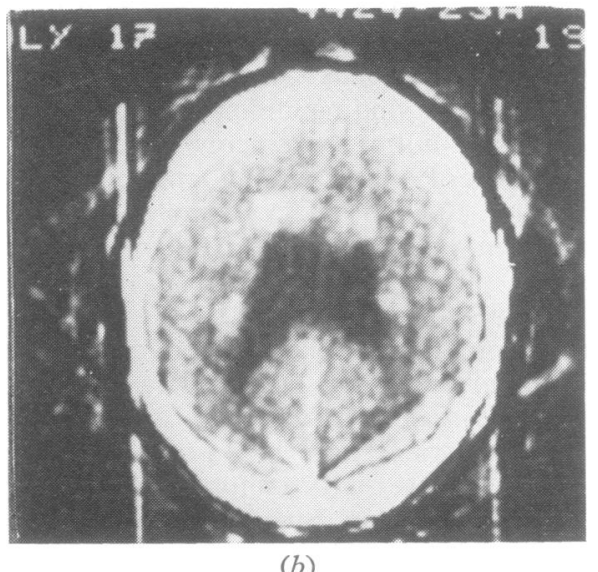

(b)

Fig. 3 Case 2. (a) Unenhanced scan showing ventricular dilatation and an area of low attenuation (12 EMI units) without mass effect near the wall of the left lateral ventricle. (b) After intravenous sodium iothalamate. There is dense enhancement of the lesion, and multiple additional enhancing lesions which could not be seen on the unenhanced scan are shown adjacent to the ventricles. later an enhanced scan was repeated; the low density area adjacent to the left lateral ventricle was still present but less prominent. No other abnormality was seen, apart from mild ventricular dilatation. A further gamma scan was normal.

After eight months the patient complained of deterioration of vision and bilateral optic disc pallor was noted. Visual acuity was $6 / 18$ in both eyes. Her hemianopia had resolved to a homonymous attention defect at this stage. Visual evoked potentials were poorly defined but suggested that there were bilateral central scotomata in addition to a left postchiasmal lesion.

Four months later she had a two week episode of weakness of the right leg which recurred after four months. She became unable to walk and was readmitted. She also gave a history of slurred speech, paraesthesiae in the hands, deteriorating vision, and hesitancy of micturition for one week. On examination she was dysarthric, drowsy, and disorientated in time and place. Visual acuity was normal but there was inattention in the right visual fields and optic atrophy. She had bilateral internuclear ophthalmoplegia and facial weakness. The jaw jerk was brisk. There was mild pyramidalo type weakness in the upper limbs and lefto leg, and severe generalised weakness in the right $\stackrel{\oplus}{\circ}$ leg. The upper limbs were ataxic. The reflexeso were symmetrically brisk with absent abdominal responses and extensor plantar responses. Thereo was a sensory level at the T3 dermatome on the right, and position sense was impaired in both feet. The bladder was distended.

On repeat examination, the CSF contained one mononuclear cell per $\mathrm{mm}^{3}$, protein $0.62 \mathrm{~g} / 1, \mathrm{IgG}$ $11.5 \%$. An oligoclonal pattern typical of multiple sclerosis was demonstrated. Visual evoked potentials were grossly abnormal; no potential could be recorded from stimulation of the left eye and that from the right was small with a latency of $127 \mathrm{~ms}$. A CT scan showed a low density area in the region of the left external capsule and a small enhancing lesion in the left parietal lobe without space-occupying effect. The ventricular dilatation was still present (Figs. 4a, b).

The patient was treated with ACTH and discharged two months later, having recovered bladder function. She was able to walk but was still moderately ataxic.

\section{Discussion}

In the two patients described, the initial diagnosis was not that of demyelinating disease, although the subsequent relapsing and remitting course with the development of typical visual evoked potentials 


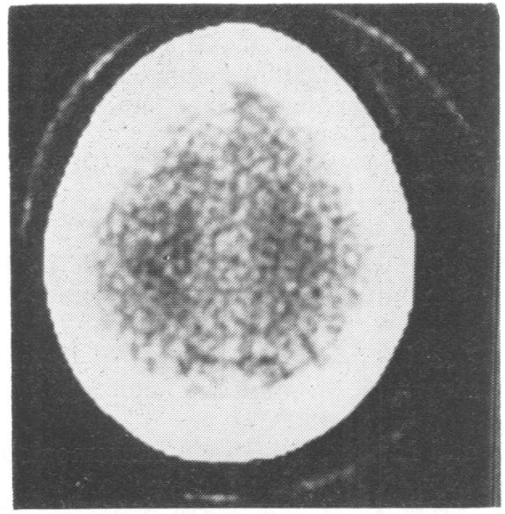

(a)

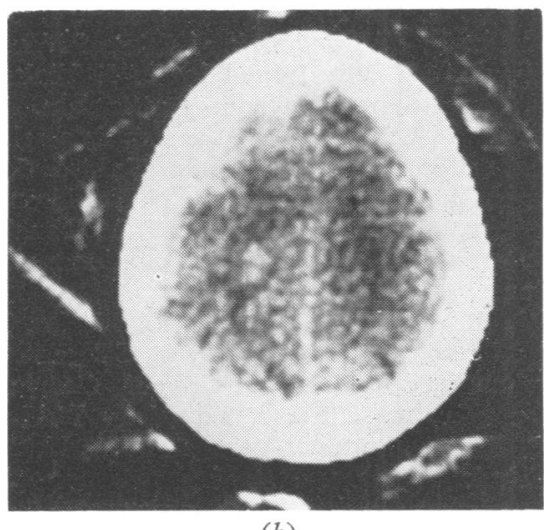

(b)

Fig. 4 Case 2. (a) There is an irregular area of low attenuation (7 EMI units) above the anterior part of the left external capsule. There is a second low attenuation lesion in the left parietal area (11 EMI units) with no mass effect. (b) After intravenous sodium iothalamate. The latter lesion shows ill-defined central enhancement.

and oligoclonal gamma globulin bands on CSF electrophoresis (Link and Muller, 1971) pointed to "clinically definite" multiple sclerosis (McDonald, 1975) in both cases. The first patient was thought to have a vascular cause for her hemiplegia at the time of presentation, probably related to the oral contraceptive pill. The recurrence one year later, together with the CT scan appearance, was highly suggestive of a tumour. Biopsy of the lesion would almost certainly have been undertaken had it not been in the dominant hemisphere. The second patient, unlike the first, had clinical evidence of multiple neurological lesions on her first admission and could be categorised as "progressive probable" multiple sclerosis (McDonald, 1975). However, visual evoked responses and CSF findings failed to support this. Furthermore, the presence of multiple enhancing lesions on the CT scan seemed more in favour of metastatic deposits than demyelinating lesions.

There are two reports of contrast-enhanced demyelinating lesions in the literature. Wüthrich et al. (1976) described multiple enhancing lesions in both hemispheres in a patient with a relapsing and remitting tetraplegia. Multiple sclerosis was confirmed at necropsy, and it is interesting to note that the areas of high density after enhancement were found to be areas of recent demyelination histologically. Older gliotic lesions were not seen on the CT scan. Cole and Ross (1977) described a patient in whom the clinical course, CSF pleocytosis, and increased gamma globulin were consistent with multiple sclerosis. Two CT scans performed within a month showed transient areas of enhancement at different sites, neither of which corresponded to the clinical findings at the time. It would seem that in these cases, and those described in this paper, the enhancing lesions were acute. This supposition is supported by the postmortem findings described by Wüthrich et al. (1976).

Clearly, not all recent demyelinating lesions cause contrast enhancement on CT scanning; if this were the case it would be a relatively common finding as scans tend to be performed for diagnostic purposes on patients with active neurological disease. Enhancement may well be a transient phenomenon during the evolution of such lesions. The mechanism of contrast enhancement in areas of demyelination is uncertain. Contrast enhancement has two different components which may be seen alone but are combined in most instances; one is intravascular, as seen in angiomas and large aneurysms, whereas the extravascular component is due to breakdown of the blood-brain barrier (Gado et al., 1975) and is seen in the acute phase of infarction. Broman (1964) demonstrated blood-brain barrier damage in multiple sclerosis by perfusing brains after death with trypan blue. It is not clear whether there was any relationship between the extent of the bloodbrain barrier damage and the age of the plaques. Lumsden (1970) commented on the discontinuity of demyelinating plaques around veins in recent lesions and suggested that this finding may be related to disturbed blood-brain barrier function. The nature of this dysfunction remains uncertain because of the paucity of ultrastructural studies on acute multiple sclerosis. Since an intravascular component of enhancement can be excluded in demyelinating disease, it must be caused by changes in the blood-brain barrier. 
Contrast enhancement of areas on CT scans can be misleading, as was the case in the two patients described here. Since enhancement appears to be transient, repeated scanning would aid differentiation of such patients from those with tumours, particularly if angiography is normal. Furthermore, the lesions tend to be localised to the white matter, have little surrounding oedema, and do not produce any space-occupying effect. The presence of enhancing areas at sites unrelated to the clinical features in cases of suspected multiple sclerosis may be helpful in providing additional evidence of multiple lesions within the central nervous system.

We wish to thank Professor John Marshall and Dr R. Ross Russell for permission to report these cases, and Dr B. Kendall and Dr J. Newsom Davis for helpful discussion.

\section{References}

Cala, L. A., and Mastaglia, F. L. (1976). Computerised axial tomography in multiple sclerosis. Lancet, 1, 689.

Broman, T. (1964). Blood-brain barrier damage in multiple sclerosis. Supra-vital test observations. Acta Neurologica Scandinavica, 40, Supplement 10, 2124.

Cole, M., and Ross, J. R. (1977). Plaque of multiple sclerosis seen on computerised axial tomogram. Neurology (Minneapolis), 27, 890-891.

Gado, M. H., Phelps, M. E., and Coleman, R. E. (1975). An extravascular component of contrast enhancement in cranial computed tomography. Part II: contrast enhancement and the blood-tissue barrier. Radiology, 117, 595-597.

Gyldensted, C. (1976a). Computer tomography of the brain in multiple sclerosis. Acta Neurologica Scandinavica, 53, 386-389.

Gyldensted, C. (1976b). Computer tomography of the cerebrum in multiple sclerosis. Neuroradiology, 12, 33-42.

Jacobs, L., and Kinkel, W. R. (1976). Computerised axial tomography in multiple sclerosis. Neurology (Minneapolis), 26, 390-391.

Link, H., and Muller, R. (1971). Immunoglobulins in multiple sclerosis and infections of the nervous system. Archives of Neurology (Chicago), 25, 326344.

Lumsden, C. E. (1970). The neuropathology of multiple sclerosis. In Handbook of Clinical Neurology, Vol. 9, pp. 217-309. Edited by P. J. Vinken and G. W. Bruyn. North-Holland: Amsterdam.

McDonald, W. I. (1975). What is multiple sclerosis? Clinical criteria for diagnosis. In Multiple Sclerosis Research, pp. 1-8. Edited by A. N. Davidson, J. H. Humphrey, A. L. Liversedge, W. I. McDonald, and J. S. Porterfield. Her Majesty's Stationery Office: London.

Wüthrich, R., Gigli, H., Wiggli, U., Muller, H. R., Elke, M., and Hunig, R. (1976). CT scanning in demyelinating disease. In Cranial Computerised Tomography, pp. 239-243. Edited by W. Lanksch and E. Kazner. Springer-Verlag: Berlin, Heidelberg, New York. 